\title{
Beyond data collection: Objectives and methods of research using VGI and geo-social media for disaster management
}

\section{Carlos Granell (Institute of New Imaging Technologies, Universitat Jaume I of Castellón, Spain)}

Frank O. Ostermann (Department of Geo-Information Processing, Faculty of Geo-Information Science and Earth Observation, University of Twente, The Netherlands)

\section{ABSTRACT}

This paper investigates research using VGI and geo-social media in the disaster management context. Relying on the method of systematic mapping, it develops a classification schema that captures three levels of main category, focus, and intended use, and analyzes the relationships with the employed data sources and analysis methods. It focuses the scope to the pioneering field of disaster management, but the described approach and the developed classification schema are easily adaptable to different application domains or future developments. The results show that a hypothesized consolidation of research, characterized through the building of canonical bodies of knowledge and advanced application cases with refined methodology, has not yet happened. The majority of the studies investigate the challenges and potential solutions of data handling, with fewer studies focusing on socio-technological issues or advanced applications. This trend is currently showing no sign of change, highlighting that VGI research is still very much technology-driven as opposed to theory- or application-driven. From the results of the systematic mapping study, the authors formulate and discuss several research objectives for future work, which could lead to a stronger, more theory-driven treatment of the topic VGI in GIScience.

\section{KEYWORDS:}

VGI; crowdsourcing; geo-social media, data analysis methods; disaster management; systematic mapping

This paper can be cited as:

C. Granell, F. O. Ostermann. Beyond data collection: Objectives and methods of research using VGI and geo-social media for disaster management. Computers, Environment and Urban Systems, 59: 231-243, 2016, ISSN 0198-9715. http://dx.doi.org/10.1016/j.compenvurbsys.2016.01.006 


\section{Introduction}

In 1941, the BBC called for people's collaboration to obtain holiday pictures and postcards of European beaches. Over 10 million of items were received ${ }^{1}$. This early precedent resembles in scale and quality of information modern responses to disasters: people generate and collect dozens of millions of media content for supporting diverse activities during all stages of disaster management (Meier, 2015). What makes the current utilization of user-generated content different is that many of the original technological limitations on the production, collection, and processing of data no longer exist. People now produce data in many ways, and many do not realize that they are constantly generating data through their own handheld devices (e.g. automated GPS traces).

The term Volunteered Geographic Information (VGI) was coined in 2007 (Goodchild, 2007) to emphasize the concept of user-generated content attached with geospatial information (e.g. location, place names) in the form of geotags or coordinates. No matter what people, services, devices, and sensors are sensing (e.g. noise, air quality), spatio-temporal context is a must to help in the understanding and interpretation of the collected data (Sagl et al., 2012). Various sources of VGI have been used in many contexts and diverse application scenarios, leading to a family of terms which highlight slightly different characteristics on the level of user participation, on whom produces the data (citizens, sensors, etc.), or on the particularities of data collection processes. Some authors have tried to create taxonomies for user-generated geographic content (Craglia et al., 2012). In this paper we continue to use the term VGI, albeit in an inclusive manner that also encompasses data that was not explicitly volunteered.

Contrary to the BBC case, where each picture was manually processed to help find the final location in Normandy, one of the determining factors today is the existence of enabling analytics technology (Chen et al., 2012) to quickly process and analyze huge amounts of data. The actual data-rich context reduces emphasis on the accuracy and exactness of data in favor of allowing some degree of inaccuracy, uncertainty and noise in return for capturing a far more comprehensive, larger set of data (Mayer-Schönberger \& Cukier, 2013). The current state of the art in data analysis techniques enables the rapid discovery of correlations in large data. More data might also help to deal with inaccuracy of individual bits of data. In this context, we assume that data analysis techniques will gain traction over data quality and precision as long as more and more VGI is captured and used in projects and applications.

\footnotetext{
${ }^{1}$ http://en.wikipedia.org/wiki/Operation_Overlord
} 
Recent works in the literature (Neis \& Zielstra, 2014; Roick \& Heuser, 2013) examine the nature of VGI itself (e.g. quality, accuracy, precision), focus on the role of the contributor (e.g. gender, motivating factors to contribute), or explore the defining capabilities in terms of reliability, documentation, and easy-to-use of current VGI sources. This study focuses on the utilization and analysis of VGI in the domain of natural and man-made disasters management. While recent reviews question current data practices on using VGI during disaster management scenarios (Haworth \& Bruce, 2015), this study takes a complementary view assuming that the detection of hidden and emerging patterns on the utilization of VGI could pave the way to advancing the VGI research field beyond its predominant focus in data collection to a level where rich spatiotemporal contexts, and advanced geospatial analysis techniques play a dominant role (Crampton et al., 2013).

In this sense, we expect that VGI research is now a stage where a body of knowledge and best practices should emerge. This is a difficult task because research on VGI is mostly technology-driven and changes at a fast pace. We aim to contribute to an ongoing consolidation by identifying important analytical trends and use patterns on the utilization of VGI, in order to shape future research and applications in the field.

To do so, we conducted a systematic mapping study that aims to find and classify the primary studies in the VGI and disaster management field. Engström and Runeson (2011) summarized a systematic mapping study as a useful tool looking "at a higher granularity level with the aim to identify research gaps and clusters of evidence in order to direct future research". In this work, we present the results of a systematic mapping study based on a sample of VGI-related studies published since the term's inception in 2007 . To do so, we first designed a classification schema that allowed us to explore systematically the set of eligible papers through a "purpose" dimension. This enables us to investigate relationships between purposes, VGI sources and analysis methods, and reveal hidden and emerging patterns to expose novel, innovate purposes for VGI beyond data collection in disaster management situations. We argue that the outcomes of the present research are valuable also for researchers in other applications domains by taking the analysis framework as reference for subsequent studies.

In particular, this systematic mapping study addresses the following questions in the context of the disaster management domain:

- Q1: Which is a suitable classification schema for VGI research to enable a systematic mapping study?

- Q2: What are the most investigated intended uses, and in what user case scenarios?

- Q3: What are the most frequently VGI data sources, and in what context/application? 
- Q4: What are the most frequently (spatial) data analyses methods, and in what context/application?

Sections 2 and 3 cover the systematic mapping review itself. We describe the methodology to obtain the set of eligible studies and the subsequent analysis for identifying main intended uses, data sources employed, and key enabling analysis methods. In section 4 , we interpret the results, and point out our own observations; whereas in Section 5 we discuss opportunities and challenges

\section{Method}

A systematic mapping study is a successful tool in research fields such as software engineering (Petersen et al., 2008) and rich web applications (Casteleyn et al., 2014). We adopt the methodology from Petersen et al. (2008) for this study. In short, the study answers specific research questions (Q2-4), related to the identification and coverage of the field of study, by first identifying eligible and primary studies, then classifying them in a newly developed schema (Q1), and analyzing the results. The following subsections detail each step of the approach.

\subsection{Search and selection criteria}

Initially, we obtained 426 papers as a result of several bibliographic search queries in major specialized and general databases engines such as ISI Web of Science, Scopus, ACM, IEEE, and DBLP, as well as thematic repositories like the Humanitarian Computing Library ${ }^{2}$. We also sought for relevant conferences and workshops for which VGI4DM was a central topic (See annex A). In order to better understand the resulting set, we performed an initial, three-phased exploratory analysis, using the following eligibility criteria:

1. Publication in scientific journals, magazines, conferences, symposia or workshops (excluding review and survey papers, editorials, comments and prefaces) with full text being accessible.

2. Written in English.

3. The title, abstract or keywords explicitly mention the utilization of VGI sources (including geo-social media) in a disaster management or crisis response context.

4. The publication was published in or after 2007, when the term VGI was coined.

During the first phase, a screening of titles and abstracts led to the removal of duplicates and those that clearly did not fulfill all eligibility criteria, reducing the set to 119 .

\footnotetext{
${ }^{2}$ http://humanitariancomp.referata.com/wiki/Welcome
} 
A second in-depth analysis of the resulting set focused on criterion 3, the relevance and use of VGI or geo-social media in a disaster management context, resulting in a final set of 59 relevant and representative papers for the systematic mapping study. These papers appear in Annex C. In total, 35 unique publication venues were identified, of which about two thirds (25) features only one publication (see Annex A). This high dispersion reflects the diversity of VGI research that spans many diverse disciplines and scientific fields. However, GIS journals are well represented in our selection and are identified as core journals in a recent bibliographic study about GIS journals (Scarletto, 2014).

Third and finally, we conducted an extensive qualitative analysis ${ }^{3}$ in order to extract and synthesize relevant data about the remaining papers $(\mathrm{N}=59)$. For each paper, we tried to answer: What is the main focus and intended use? What kind of data do the authors employ for their study? What data analysis and visualization techniques do the authors utilize? Do the authors compare or integrate their results with official/reference datasets? What is the use case or scenario? Who are the target user(s)? As a result, we extracted a list of 30 variables grouped on four thematic clusters:

- Article's bibliographic details: unique identifier; title; year of publication; the name and type of the publication venue, keywords as they appeared in the paper; DOI and abstract.

- Article's focus and intended use: the main category of a paper, its focus, and intended uses (the combination of these three variables contributed to the build-up of the classification schema described in the following section); the most relevant application domain (natural disaster, man-made disaster, both; use case or scenario (e.g. floods, earthquake, riots, etc.); a concrete disaster, if any (e.g. 2010 Haiti Earthquake); and type of stakeholders or end users if explicitly mentioned.

- Data sources employed: (geo-)social media/VGI sources used; additional comments/notes on how the VGI sources were used; use of official/reference data, and if so, which ones.

- Analytical strategy and analysis methods employed: the type/strategy of analysis conducted; the methods and/or protocols used for data collection; the manual data analysis and crowdsourcing methods, automated data analysis methods, or specific geospatial-related methods used during the data preparation phase; the network analysis techniques, applied statistics methods, or specific geospatial analysis used

\footnotetext{
${ }^{3}$ The resulting data set from the analysis, plus documents explaining all the variables of the data set, along with the subsequent data analysis (R scripts) for Section 3 are publicly available in the following Github repository: https://github.com/cgranell/paper-vgi-science
} 
during the analysis phase; the geospatial methods used for visualization purpose; and whether or not the paper describes a tool.

\subsection{Classification schema}

The resulting classification schema addresses Q1 and groups the studies into a taxonomy of three levels: Main categories, focus, and intended use. The main category refers to the overall context of the research, the focus captures the main objectives of the study within each category, and the intended uses are meant to express finer aspects of each focus. For instance, location extraction and classification is a concrete use of a paper whose overall focus is data preparation or handling. Categories and focus are not exclusive since a paper may belong to various categories or may have more than one focus.

The classification schema is based on two inputs: First, from discussions between the authors and therefore based on their domain knowledge. Second, as a result of the exploratory analysis (Section 2.1) by identifying the research context and main concepts that reflected the main contribution of each paper. By doing so, we identified three main categories: data-centric, human-centric, and application-centric. Given the focus on disaster management and response, the application-centric category currently consists only of crisis management as single instance.

In addition, keywords were analyzed to elicit information about the main focus of each paper. The process to extract and define foci was iterative by adding, deleting and merging them over the course of the analysis as we were getting a better understanding of the concrete contributions of the papers. The third level of the classification schema was achieved by identifying finer intended uses through a detailed analysis of the papers. The combination of categories, foci and intended uses gave us an initial classification schema to categorize papers and provided an overview of the finer intended uses of the utilization of the VGI for disaster management.

The data-centric category contains studies that mainly concern the management or handling of (big) VGI4DM streams. It includes studies whose general goal is to transform raw data into useful and relevant data, i.e. "processed data" ready for subsequent analyses. Examples of data processing and transformation tasks are cleaning and filtering, annotation, clustering, aggregation, and contextualization, which form the basis for the definition of the set of sub-categories:

- Data preparation: Cleaning, filtering and extraction of extract relevant data from raw data sources.

- Data contextualization: Data enrichment with contextual information (e.g. from external sources). 
- Data quality assessment: Assessment of the veracity and reduction of bias of the data.

- Data preservation: Strategies to long-term social media data storage to permit future studies on past data.

- Data policy: Various aspects such as data access rights, terms of service, copyright and the like about VGI and social data.

By drilling down further, we identified a set of intended uses of VGI data for each subcategory within the data-centric category, which are summarized in the figure 1.

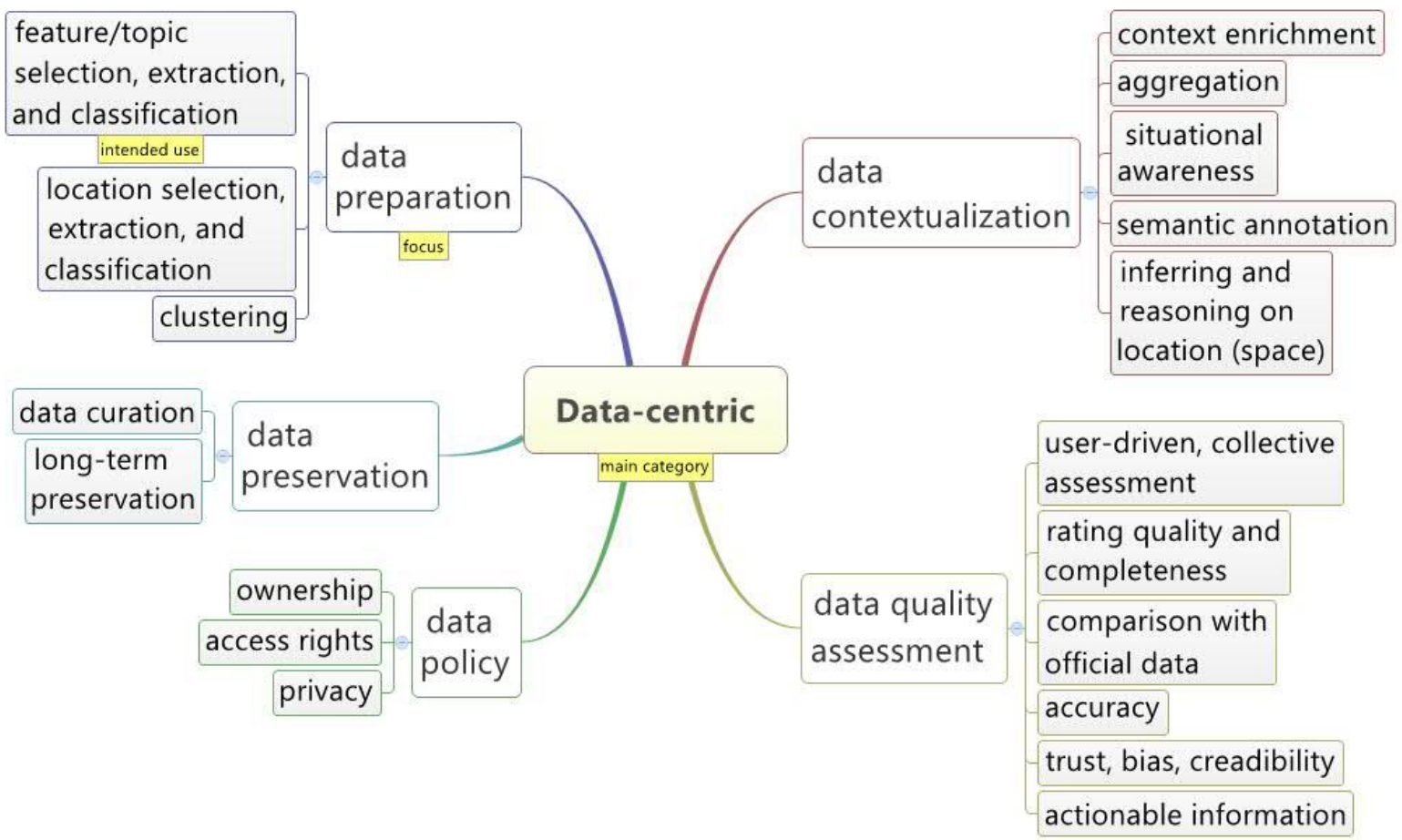

Figure 1: Data-centric category (first level), focus (second-level) and intended uses (third level) in VGI-related studies for the review

The human-centric category includes studies that focus on people as central theme and that explore human activities and social digital footprints from VGI sources. Human-centric papers investigate a wide range of important social phenomena, including demographics, social relations, interactions and behaviors. The foci are:

- Human activities: Any kind of human activities which may be the target of VGI analyses.

- Human mobility: A special type of human activity related strongly to location, thus deserving its own sub-category.

- Human relations: How people relate to others, and how they are grouped together in similar communities or clusters.

- Human perceptions: The level of awareness or public perception of a theme or topic, i.e. how people feel particular disaster events and situations. 
Similarly to the data-centric papers, we identified intended uses of VGI for each focus within the human-centric category, as illustrated in figure 2.

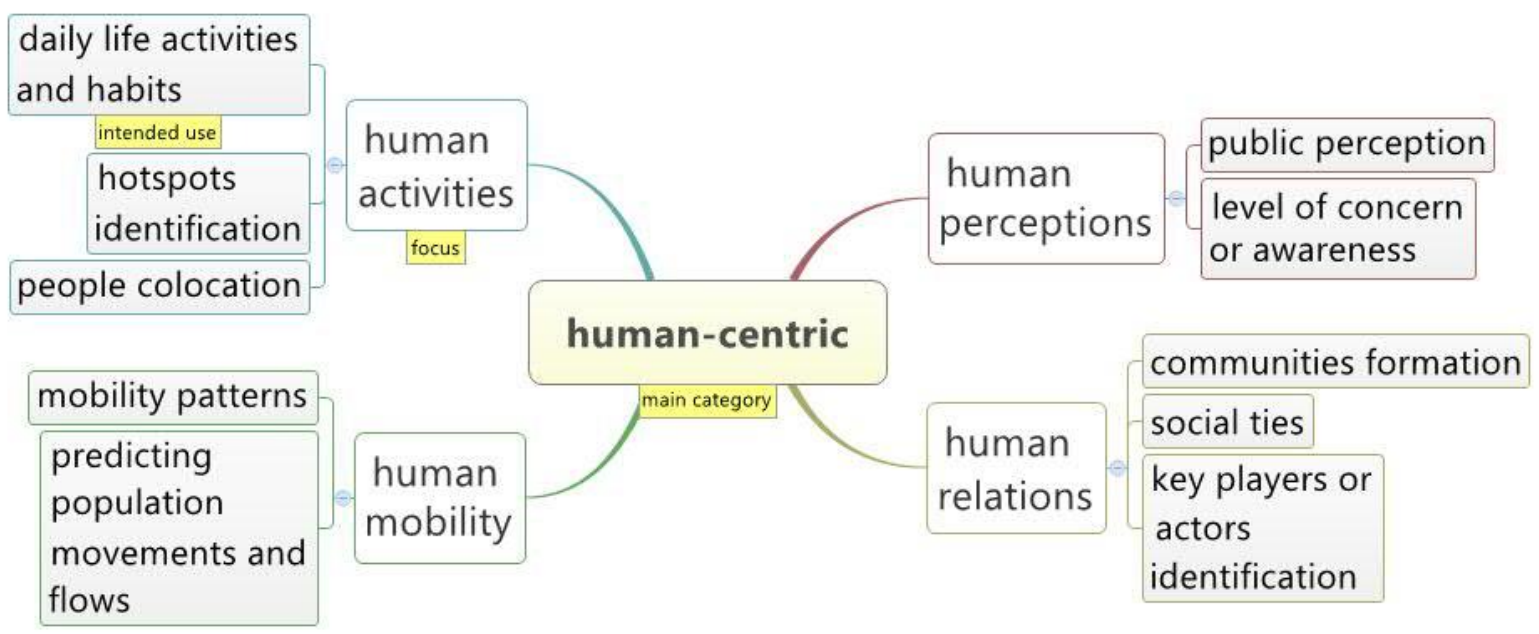

Figure 2: Human-centric category (first level), focus (second-level) and intended uses (third level) in VGI-related studies for the review.

The application-centric category comprises studies that are concerned with the use and application of VGI to the particular case of natural disaster, e.g. earthquakes, tsunamis, floods, tornados, and man-made events such as social movements, protests, riots, and so on. We arrived at the following self-descriptive sub-categories for the application-centric category, which fit the four phases of crisis management during the outbreak of natural or man-made events (Cutter, 2003) plus public health issues:

- Crisis detection and prediction,

- Crisis monitoring,

- Crisis recovery and response,

- Crisis coordination and organization, and

- Crisis health.

Finally, we also identified intended uses within the application-centric category (Figure 3). 


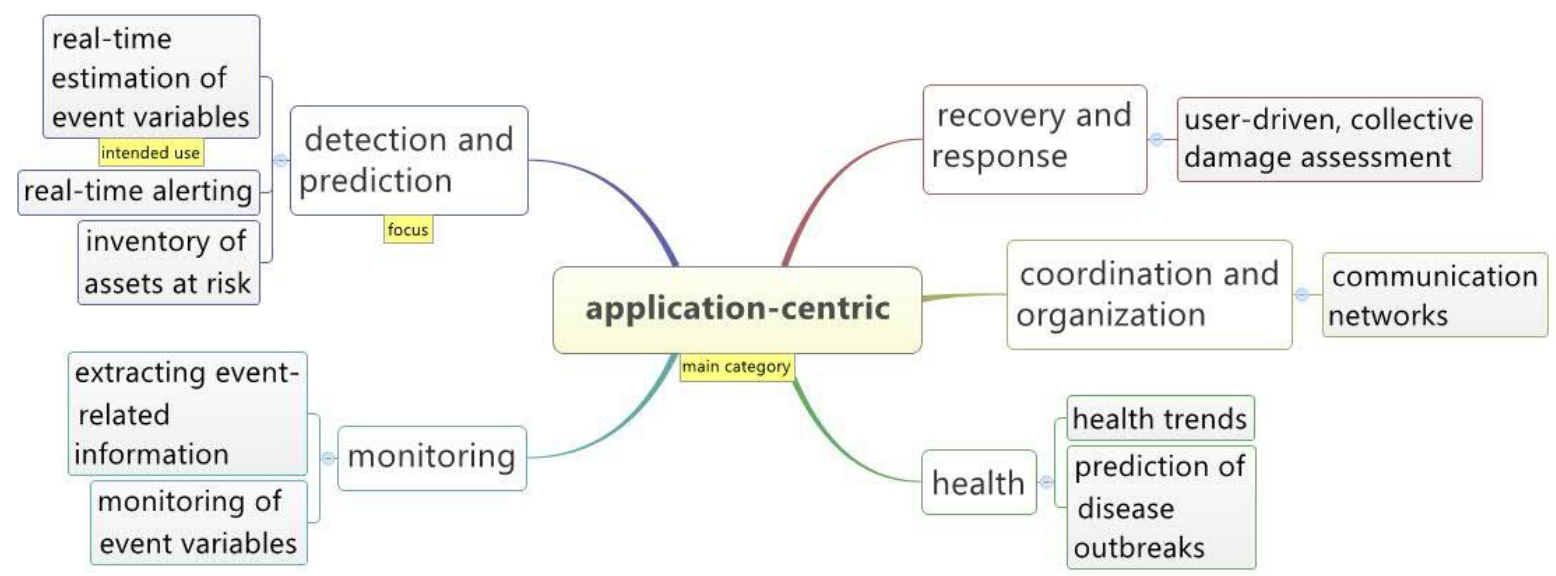

Figure 3: Application-centric category (first level), focus (second-level) and intended uses (third level) in VGI-related studies for the review.

\section{Results}

The section presents the results of the systematic mapping review. We first map the set of eligible studies $(\mathrm{N}=59)$ to the classification schema (Q1). This exercise allows to address Q24, with each question being dealt with in a separate subsection (Sections $3.1-3.3$ ).

The three tables in Annex $B$ result from mapping the outcomes of the exploratory analysis into the classification schema described earlier. They correspond to the three application categories, i.e. data-centric, human-centric, and application-centric. Each table relates each focus with the set of intended uses extracted from the papers, and also gives examples of analysis methods used, which will be explored in detail in the following sections.

\subsection{Focus and intended use}

The definition of an initial research question is essential to drive, focus, and contextualize a study. Every analysis aims to answer a given question, and each question may require different analysis methods and strategies to address it (Leek \& Peng, 2015a). The focus and intended use of the study therefore should determine the analysis methods, tools and the set of data sources.

To explore the focus and intended use of the studies, we first observe the overall trend of the entire set of papers. The number of papers tagged as data-centric (41, or $53 \%$ ) outnumbers the other two categories (24 as application-centric: $31 \% ; 12$ as human-centric: $16 \%$ ). As mentioned, a paper can belong to more than one main categories. 5 papers are labeled as data-centric and human-centric, and 13 papers are data-centric and applicationcentric. This indicates that these papers make significant contributions to both categories. For example, these studies often focus on data handling and then apply the results to either 
application- or human-centric use cases. There is no overlapping between human-centric and application-centric categories.

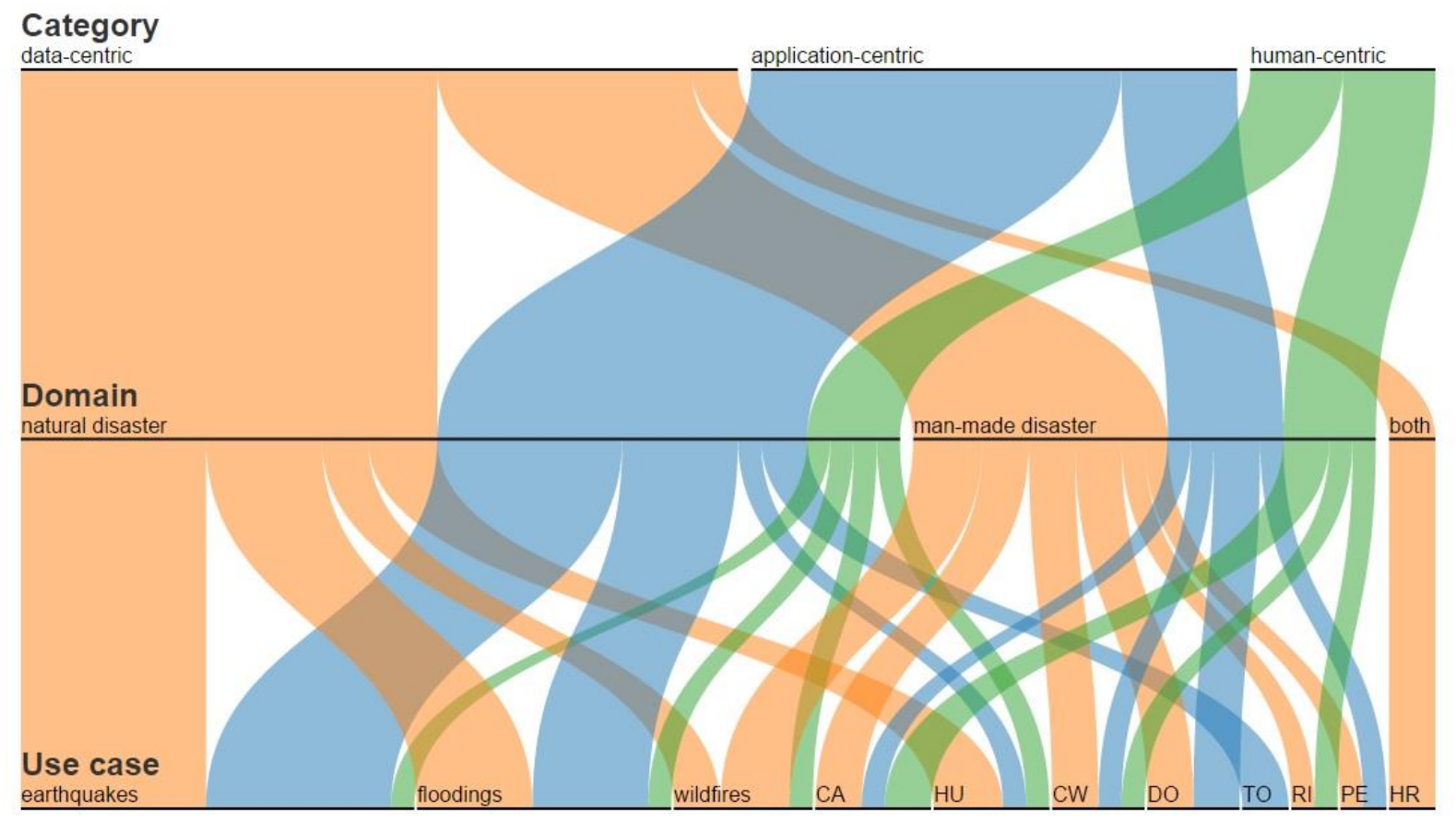

Figure 4: Parallel set visualization to illustrate how the set of papers are related considering the main category, application domain, and use cases variables. Use case abbreviations are defined as: CA stands for crowded areas; HU hurricanes; CW civil wars; DO disease outbreaks; TO tornados; RI riots; PE protest events; and HR hurricanes and riots.

The parallel set visualization in Figure 4 illustrates the relation among main categories (identified by color), application domains (natural disaster, man-made disaster, both) and use cases. First, the total number of papers in the natural disaster domain (63\%) doubles that of those in the man-made disaster domain (33\%), with $4 \%$ of the papers addressing both domains. In particular, natural disasters are predominant in data-centric and application-centric related studies, while man-made and natural disaster domains turn out to be evenly distributed in human-centric studies. Based on the set of analyzed studies that specified application domain and use case, earthquakes and flooding are by large the recurrent use case in application-centric studies and data-centric studies, followed by wildfires, and hurricanes. Most use cases also correspond to those found by Horita et al. (2013).

We observe that a significant proportion of studies within the data-centric and humancentric categories do not explicitly indicate an end user or stakeholder, and some studies do not specify a use case at all. Conversely, application-centric studies do often indicate stakeholders, i.e. vested users interested in the results and/or usage of the application. In such cases, emergency managers are mostly identified as target users or stakeholder. 
With such preponderance of data-centric studies, it is worth looking more closely at the focus and concrete intended uses. Figure 5 shows the frequencies of intended used (y axis) grouped by focus (color). At first glance, the frequencies of intended uses are very unevenly distributed, with the data preparation focus and associated intended uses the most frequent. This suggests that many studies concentrate on the selection, extraction and classification of features of interest (i.e. topics, theme, location, places, etc.) from source data streams (See Table 1 in Annex $B$ for examples from literature). Activities related to the handling and preparation of VGI4DM are still the predominant goal of many studies.

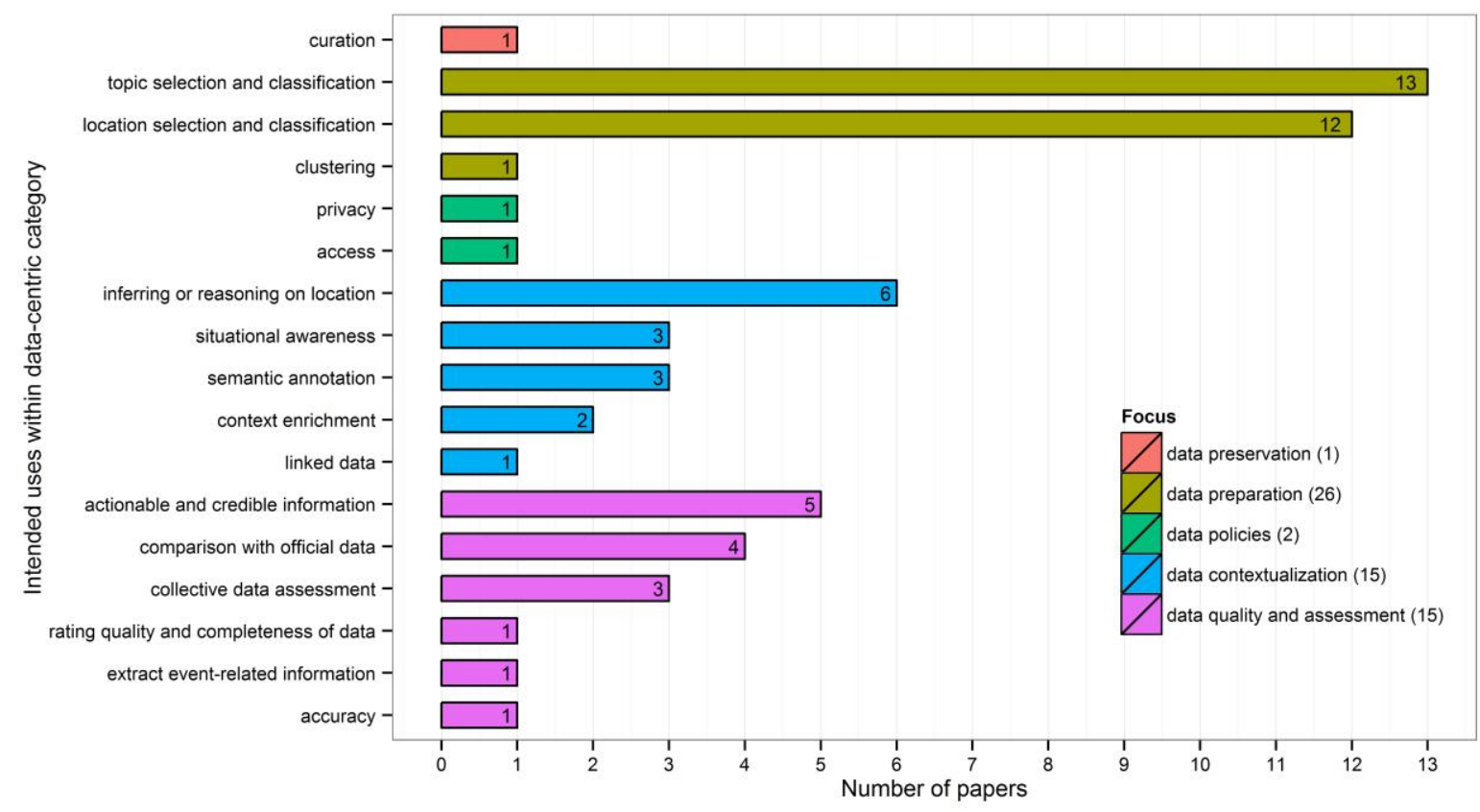

Figure 5: Intended uses by focus within the data-centric category. Note that a single paper can have more than one intended use.

An equal number of papers address issues of data contextualization and data quality and assessment. Data contextualization refers to annotating and enriching bits of data extracted from VGI4DM sources with contextual information that often originates from alternative data sources. Its aim is to enhance situational awareness and contextual narratives. This shows that some studies in the VGI4DM research field are moving from extracting and classifying bits of data (data preparation focus) towards more elaborated, knowledge-intensive uses. Intended uses within data contextualization include capturing place-time-theme narratives for situational awareness purposes (Rogstadious et al., 2013; McEachren et al., 2011; ), annotation and augmentation of VGI data with spatial semantic descriptions (Abel et al., 2012; Ortmann et al., 2011; Schulz et al., 2012;), enrichment of VGI data with alternative spatio-temporal contextual data (Spinsanti \& Ostermann, 2013; Davis et al., 2013; De Longueville, Luraschi et al., 2010; Horita et al., 2015); inferring and enriching one's location by 
combining additional data sources (Davis et al., 2011; Diakopoulos et al., 2012; Ikawa et al., 2012), and integration of heterogeneous geospatial ontologies and concepts (Zhang et al., 2014; Intagorn et al., 2010).

The utilization of VGI4DM for data quality and assessment has two types of studies: those that try to assess the quality, relevance and trustworthiness of VGI4DM data by comparing it with other data sets (e.g., Foody, 2014; Spinsanti \& Ostermann, 2013; Power et al., 2013; Panteras et al., 2015; Kent \& Capello, 2013; Antoniou et al., 2010; Camponovo \& Freundschuh, 2014), and those that use people as quality assessment tools (e.g., Rogstadious et al., 2013; Ortmann et al., 2011; Barrington et al., 2012; Popoola et al., 2013). Both represent different but potentially complementary approaches to verify the quality of VGI (Goodchild \& Liu, 2012). Hybrid strategies that combine methods to integrate VGI4DM and official data sets examined by large groups of volunteers are still rare but seem to be a promising niche to explore in the future, especially during the aftermath of disaster events.

Finally, foci relative to data preservation and policies seem to attract less attention from the VGI4DM research community. As seen in Section 2.1, most eligible studies have been published in computer science venues, and so the predominant focus on technology-intensive research (data preparation, contextualization and quality assessment) dominates over other topics from the social sciences community.

Figure 6 shows the foci and intended uses within the human-centric category. Most studies explore human activities, with human mobility being a special case since location is a dominant, central feature in such cases. For example, determining and predicting peoples' movement and density in and aftermath of disaster events or social events (protests, riots, etc.) is widely explored (Bengtsson et al., 2011; Chen et al., 2011; Wirz et al., 2013). Similarly, human activities like the identification of people's daily habits (Girardin et al., 2008; Crandall et al., 2010; Andrienko et al., 2013) and topic of conversations (McNamara \& Rohner, 2012) have been also investigated in disaster emergency situations. An important aspect in disaster situations is the characterization of the affected communities. Revealing social relations and/or ties between members of such communities may help response agencies to discovery community leaders who have predominant roles within their communities (Cheong \& Cheong, 2011).

Even though other studies with a human-centric focus have been applied to varied use case scenarios other than disaster managements, they still remain of interest in such cases because of their methodological approach. Inferring co-occurrences of people in time and space, also known as people co-location (Crandall et al., 2010), travel patterns and recommendations (e.g., Girardin et al., 2008), and commuters habits (Zatlz et al., 2013) are of 
value to response agencies and emergency managers when it comes to evaluate all factors for taking decisions.

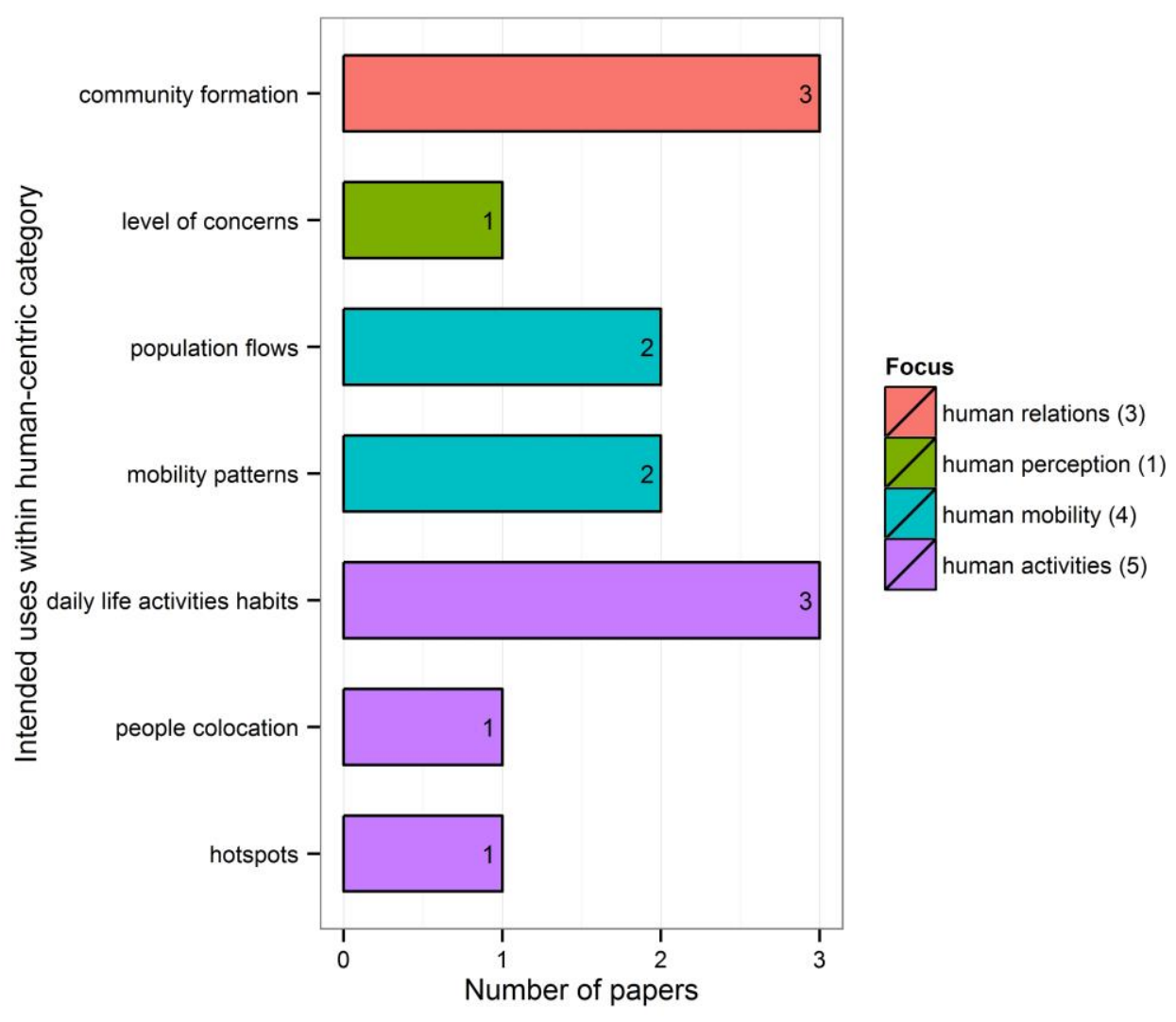

Figure 6: Intended uses by focus within the human-centric category. Note that a single paper can have more than one intended use.

For the third category, application-centric papers, social media services and VGI tools become powerful communication tools to disseminate information to the public (e.g., for alerts), to gather information from the public (e.g., crowdsourcing), and to coordinate actions among crisis management professionals. Figure 7 shows that most studies have investigated the use of VGI4DM as an early warning and detection mechanisms (e.g. Crooks et al., 2013; Earle, 2010; Robinson et al., 2013; Schelhorn et al., 2014), and for monitoring purposes to extract event-relevant information (e.g., Rogstadius et al., 2013; Liang et al., 2013; Budak et al., 2013; Poser \& Dransch, 2010; Horita et al., 2015). However, there is also a significant effort on collective, user-driven damage assessment during the response and recovery phases (e.g., Curtis \& Mills 2012; Santamaria et al., 2013; Corbane et al., 2012; Barrington et al., 2012; Lue et al., 2014). The variety of intended uses extracted from the set of studies shows that VGI collected and captured over the course of a crisis event is a valuable source of information for emergency agencies and managers to carry out detection, monitoring and assessment activities as well as to take actions for recovery and rapid response. 


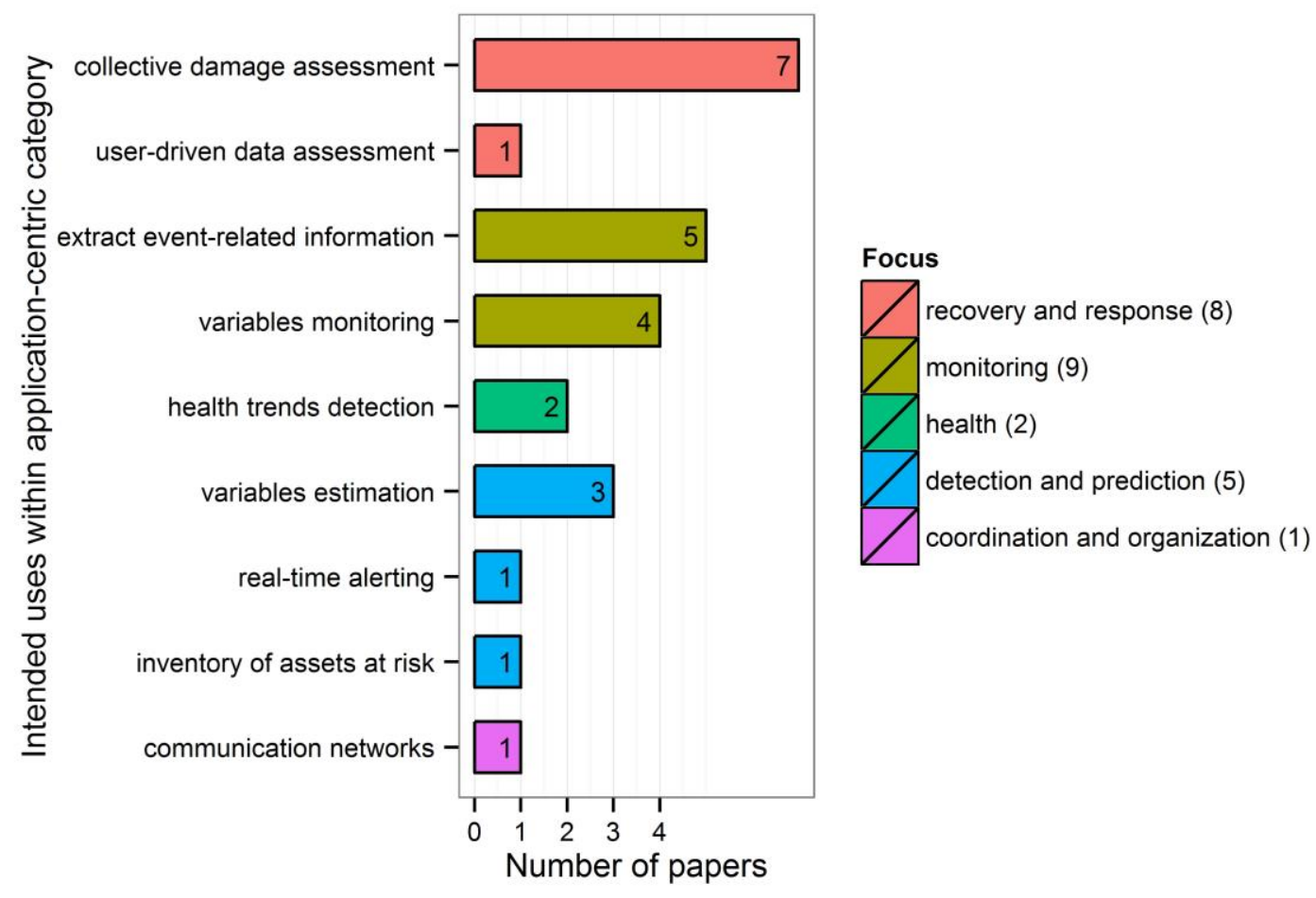

Figure 7: Intended uses by focus within the application-centric category. Note that a single paper can have more than one intended use.

\subsection{Data sources}

A critical step for any scientific inquiry is the identification and choice of relevant data sources meeting the study. Developing or using well-documented procedures and automated methods to collect data from varied sources fosters the reproducibility of VGI-related studies of interest by other researchers (Ostermann \& Granell, 2016).

Figure 8 illustrates that the overwhelming majority of studies use Twitter as data source. Some studies choose other data sources because of the special characteristics of their context and intended use, e.g. GPS data from mobile phones to trace people movements after crisis events (e.g. Bengtsson et al., 2011; Wirz et al., 2013), spatial video for assessment and monitoring of the affected environment during post-disaster phases (e.g. Curtis \& Mills, 2012; Lue et al., 2014), detection of road blockage using Open Street Map and airborne light detection and ranging (LiDAR) data (Liu et al., 2014), and unmanned aerial vehicles for quick mapping purpose (Santamaria et al., 2013). Despite these examples, Twitter clearly is the primary (and often the unique) data source for disaster management use cases. 


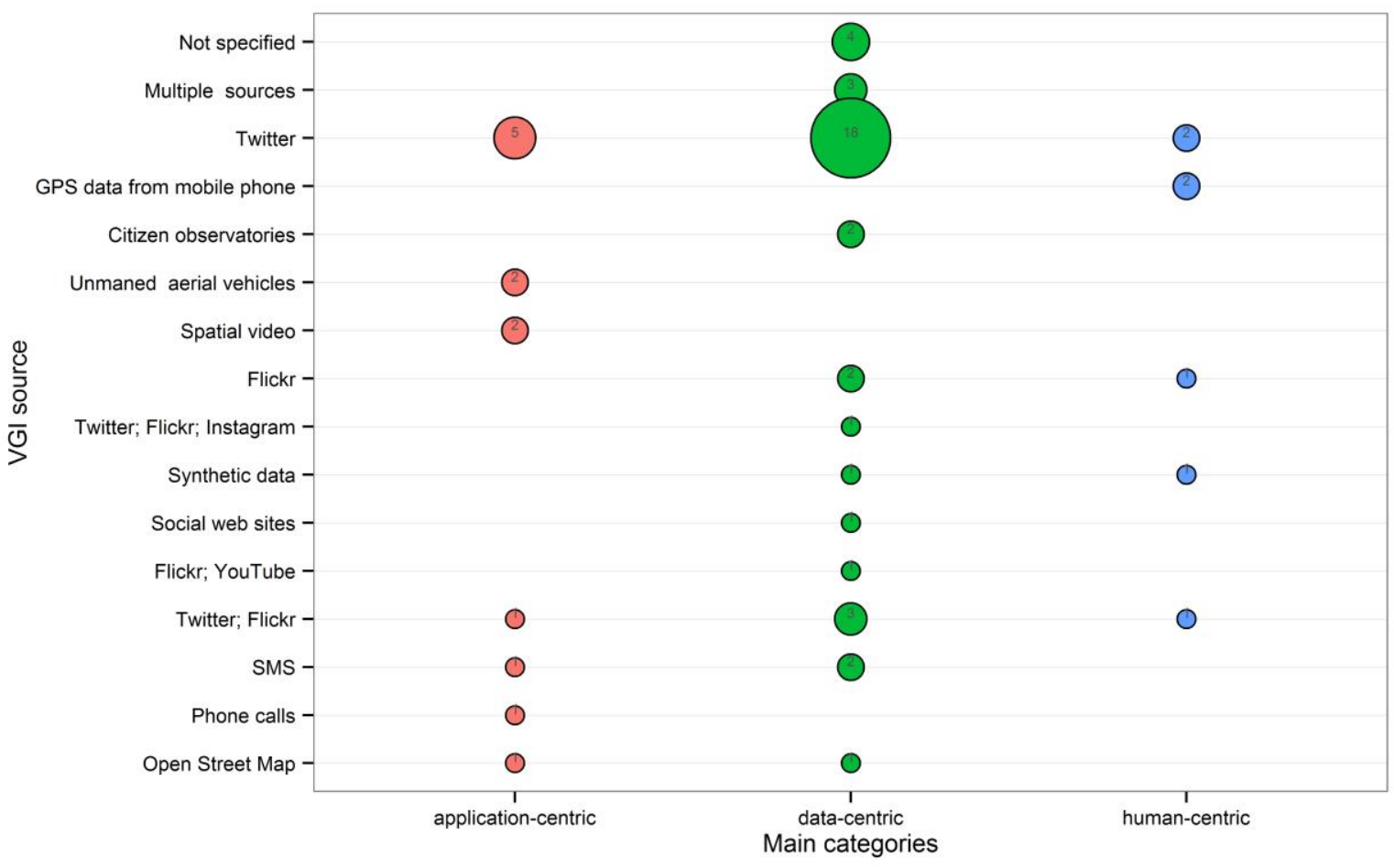

Figure 8: VGI sources employed by main category.

The use of Twitter as a unique data source comes at the expense of having only few studies using two or more VGI sources simultaneously or combining or integrating VGI sources with other official data sets. The degree of Twitter use varies depending on the focus. For example, Twitter is the predominant source in almost all data-centric studies, especially in data preparation, contextualization, and quality assessment, with 18 cases in total as unique source but 25 if combined with other sources, followed by Flickr with 7 occurrences, both alone and in combination with other sources. Twitter is exclusively used for detection and prediction, and monitoring foci in the application-centric category. This is due to most studies focusing on earthquakes, where Twitter practically is the only feasible VGI source for early detection. However, Twitter is not so widely used in recovery and response foci in favor of other data sources such as spatial video, UAV, and phone call data, which may provide strong evidence (e.g. in-situ images) of the current situation of an affected area during post-disaster stages.

Surprisingly, Twitter is less frequently used in human-centric studies. In fact, this category presents a scarce variety of data sources but mostly used in isolation. For example, Twitter, Flickr, and GPS data from mobile phones are examples of unique sources used in human-centric studies. In fact, GPS data (2 cases) is exclusively used in studies relative to the human mobility focus. 
Finally, we cannot observe a distinctive feature on the use of VGI4DM sources in combination with official data except for all of the studies classified in the data contextualization and data quality and assessment foci that use additional official sources.

\subsection{Analysis methods}

The definition of an initial research question in a data analysis study is fundamental because it determines the most suitable overall analysis approach or strategy, which in turn should determine the pool of analytical methods to employ. Many studies in our set do not explicitly define an initial scientific question that shapes the analysis strategy and guides the subsequent data analysis. They briefly state vague or broad objectives such as to improve decision making for disaster management and to enhance situational awareness, but do not operationalize these vague research questions into a suitable strategy for data analysis. This lack of information about the research questions and target users of the analysis made it difficult in many cases to identify or interpret the analysis strategy. From the literature, we distinguish the six broad analysis strategies or approaches: descriptive, which describes or summarizes data sets; exploratory, which tries to find relationships or patterns and develop objectives for follow-up studies; methodological, where authors propose and test methods and workflows; inferential, which aims to generalize results from small data samples to the population; predictive, which utilizes some variables (predictors) to predict values for another object variable (outcome); and causal, which tries to find out the conditions under which associations and correlations amid variables can be interpreted as causality.

Almost all of the examined studies have been categorized as descriptive or exploratory. Descriptive studies summarize data via descriptive statistics (e.g. mean, median, standard deviation, quartiles, etc.) and plots (histograms, box plots, etc.) without deeper interpretation. More than half of the studies are of exploratory nature. They often build on a descriptive analysis to discover patterns and correlations between observations, help interpret statistic coefficients and errors, and measure data uncertainty. Within this group, well-known Natural Language Processing (NLP) and Machine Learning (ML) techniques are used for text analysis, information retrieval, and in general for data preparation purposes (i.e., filtering, classification, clustering etc.). This includes for example activities to compute word frequency, word disambiguation and to extract and identify entities of features of interest like place names using named entity recognition tools. Also supervised and unsupervised classification and clustering techniques are applied in order to group relevant entities (e.g. emotions, place names) and detect patterns. These techniques often required fine-tuning to handle the extremely short and unstructured content of the text field in Tweets. 
A few studies may be regarded as inferential or predictive analysis for quantifying discoveries beyond the dataset in hand and predicting measurements respectively. Examples are probabilistic models (Crandall et al., 2010), predictive models (Bengtsson et al., 2011; Chen et al., 2011; Wirz et al., 2013), and regression analysis (Wirz et al., 2013; Panteras et al., 2015; de Alburquerque et al., 2015; Kent \& Capello, 2013). Causality is absent in the studies analyzed.

With regards to the pool of geo-analysis methods, many papers customize well-known NLP and ML techniques to support geo-parsing and geo-location. Contextual information such as one's current and past messages, one's profile, and locations of one's relations prove useful (Ikawa et al., 2012). Some studies analyze spatiotemporal patterns using density surface maps (i.e. heat maps) and by computing spatial distances (Manhattan, etc.), and often produce web map-based visualizations to show the results of their experiments (e.g. Stefanidis et al., 2013; Girardin et al., 2008). Others have applied social network analysis (SNA) techniques to study the network structural properties as well as propagation and diffusion models as a result of transforming interrelated social data into network graphs (e.g., Crandall et al., 2010; Conover et al., 2013). Indeed, SNA is a versatile tool as it allows constructing different networks from the same data set to subtly study distinct human behaviors and relations. For example, Cheong \& Cheong (2011) created one network composed of nodes (or vertices) representing Twitter users and edges (or links) representing responses to particular tweets of Twitter users, and another network containing those Twitter users and linked resources (i.e., referenced by links from tweets) as nodes forming then a bimodal or bipartite networks.

For the application-centric category, we found a richer set of analysis methods than in the other two categories. For example, in the detection and prediction focus there exist more advanced models such as a geo-spread indicator for detecting quakes (Robinson et al., 2013; Earle, 2010), and a quake response model as a function of distance from epicenter over time (Crooks et al., 2013). Within the monitoring focus, examples are density and buffer-intensity calculations (Liang et al., 2013), Kriging regression (Poser \& Dransch, 2010), computation of a composite "hazard" index (Horita et al., 2015), and the Geo-scope algorithm (Budak et al., 2013) for detecting geo-correlated trends from VGI sources. SNA-related techniques such as link analysis (Conover et al., 2013) are widely used within the coordination and organization focus.

\section{Discussion}

In this section, we revisit the four research questions, synthesize the results from the previous section, and discuss implications of our findings. 
The classification schema (Q1) proved useful and provided sufficient detail to capture the various aspects of the investigated papers. Some of the schema's intended uses are sparsely populated, suggesting a merging to create fewer categories. However, this sparseness is also an indicator of current research foci (and their absence), hence it shows the richness (or lack thereof) in current research, and suggests future research topics - one of the original objectives or motivations of this study. The data-centric and human-centric categories can be re-used for exploring other applications than emergency management in conjunction with an extension and/or adaptation of the application-centric category's foci and intended uses. To complement our manual analysis and reasoning that shaped the classification schema, it would be desirable to use text mining methods over the abstracts of all papers to check whether a significant proportion of the terms we used in the focus and intended use level are explained (i.e. are highly correlated) by the most frequent associations of topics from all abstracts

Concerning Q2, focus and intended uses, Figure 9 shows that even after several years, there is still a clear overall emphasis on data-centric research. This is surprising given that our literature is based on a search with a clear application context of disaster management. It suggests that there is still a need to understand VGI4DM collection, processing and characteristics. An overall data-centric emphasis is to be expected for earlier publications when researchers were struggling to handle the volume and diversity of VGI sources. However, one could expect an increasing share of studies on human- or application-centric themes over time.

Other important activities such as data contextualization and, quality assessment have received less attention from the research community than data preparation. Spinsanti \& Ostermann (2013) proposed to enrich social data on forest fires with additional geographic context information (from external official sources) based on the location identified in VGI items. Contrasting information from VGI sources with information from official data sources (e.g. demographic, environmental observations, transport, etc.) may be certainly a way forward to discover new insights, correlations and patterns. When top-down data (official, authoritative, "objective" data) and bottom-up data (user-generated, "subjective", social data) are properly combined, the resulting contextualized data should become more precise and actionable for being used in data analyses. For example, Schade et al. (2013) have explored the use of the OGC Sensor Web Enablement (SWE) standards (OGC SWE, 2015) to model and combine VGI sources, thus converting them in a timely and valuable source of information. Other authors (Crampton et al., 2013; Roick \& Heuser, 2013; Tsou \& Leitner, 2013) as well call for further research in data contextualization and integration (especially with other official and ancillary data sources), data assessment and quality, but also data ownership and privacy. The tendency on data preparation seems to decline the past two years (Figure 9); studies 
dealing with data contextualization and quality assessment issues clearly outnumber those on data preparation tasks.

Nevertheless, coupling structured and unstructured data from many different data sources still remains challenging for several reasons: the difficulty to manage distinct protocols for data collection; the need to integrate data sets of different provenance, coverage, granularity and complexity; and the time constraints of a disaster management context. $\mathrm{A}$ potential solution could be a common data model that could be directly applied to multiple VGI sources for improving subsequent geospatial analysis (Kalanteri et al., 2014). To this end, Croitoru et al. (2013) proposed a data model composed of source-dependent and sourceindependent components. While source-dependent components refer to bits of information present in particular VGI sources, source-independent components aim to identify common information that span several sources. 


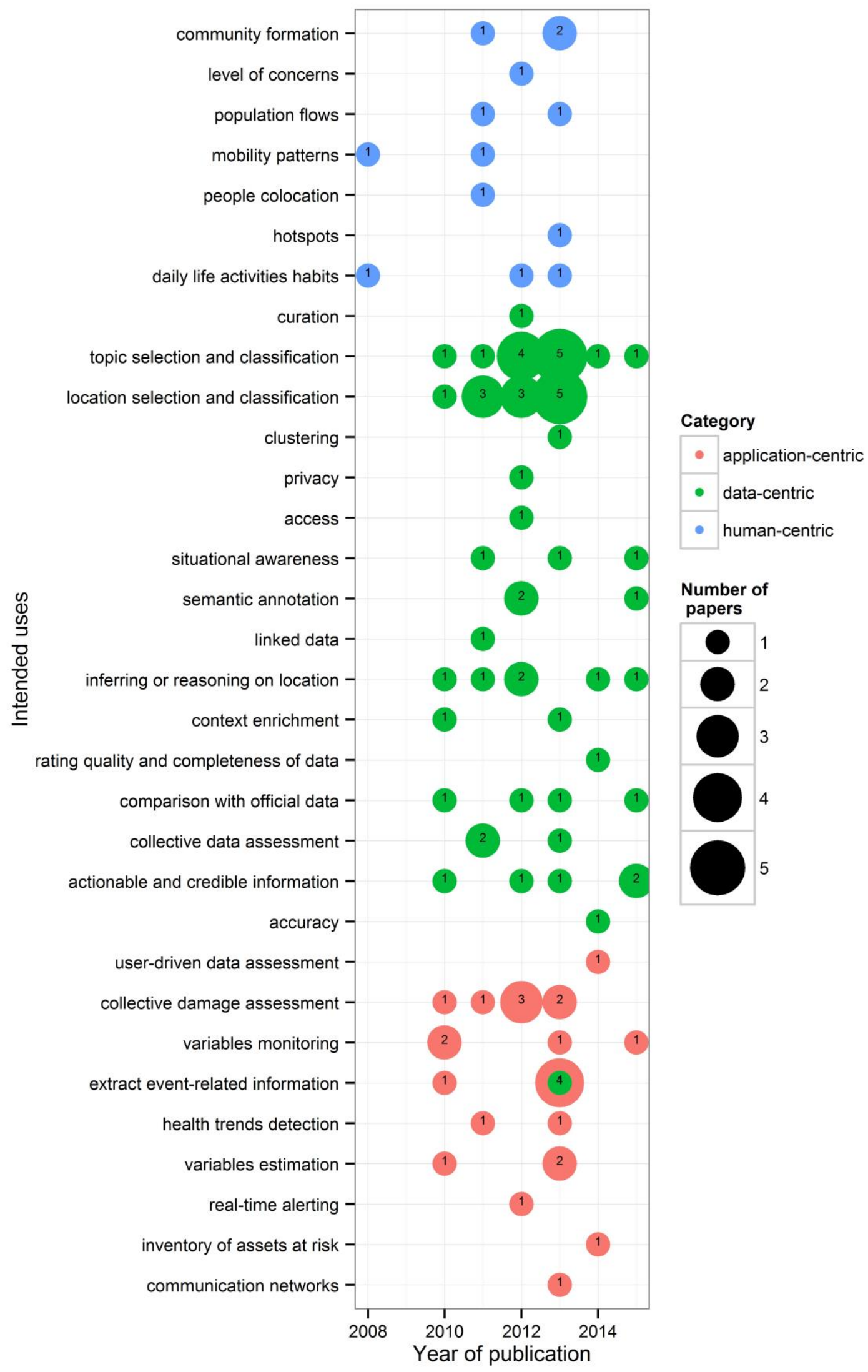

Figure 9: Intended uses over time by main category 
There is a notable scarcity of studies on data policies, ownership, data rights, privacy, and ethics. One possible explanation could be that, although important for leveraging VGI in disaster emergency contexts in the long term (Meier, 2015), the technology-driven research community might perceive them as less technology-intensive and thus of secondary concern. This would be unfortunate, since ensuring privacy during data collection is crucial. The simple assumption that anything posted publicly on social networks is meant to become public is insufficient, and a more detailed and careful assessment is necessary (Crawford and Finn 2015). Mechanisms to ensure privacy for gathered data are required (King, 2011), as well as data governance to clarify who owns the data and defines its limits of use.

For 3-4 years after the term VGI had been coined in 2007, only a few "practical" results were published apart from position/vision papers. From 2011/2012 onwards, this changed somewhat (Figure 9). The increase of application-centric studies runs in parallel with an increase studies in data-centric themes, which in turn focus on extracting and parsing location from VGI sources. This suggests that application-centric studies depend strongly on a solid substrate of operational tools to conveniently extract, parse, classify and process location from VGI sources. This fact is particularly important because it demonstrates that location is vital context information to correctly interpret, analyze and reason on VGI4DM streams, and that geospatial analysis methods and tools are cross-cutting to several domain applications, and in consequence should be considered among the core building blocks of the social media analysis field.

Regarding Q3 (data sources), the most used data source is Twitter, and this trend has even increased over the past years, as also suggested by Steiger et al. (2015). As unique source, the benefits of Twitter are public access to large data sets in the order of millions of pieces of content (although the free search API has limited access to historic data), an easyto-use and well-documented API, structured meta-data and -though unstructured-content in the form of microposts that allow researchers to apply well-known techniques to process, extract, and mine text. Another positive aspect is the "option value" of Twitter data according to Mayer-Schönberger \& Cukier (2013), which is the ability to use the data for novel, not anticipated purposes different from the primary usage. The primary purpose of Twitter data is personal communication. The studies analyzed here, even though they represent a small part of the spectrum of possible applications based on Twitter data, show the value of using Twitter data in a broad range of secondary, novel purposes. The preponderance of Twitter also comes with challenges and threats to the potential validity of results. There are several biases to consider when using social media data in general and Twitter in particular. First, there is a clear user bias, i.e. the user base is not evenly distributed across countries and socio-economic groups (Meier, 2015). Further, social media content is strongly influenced by short-lived 
feedback loops (Shelton et al., 2014). This tendency is aggravated by the possibility of users exaggerating and distorting events when posting information in social network sites like Twitter. For example Rutherford et al. (2013) demonstrated some evidence that studies based exclusively on social media data for their analyses carry a degree of bias which consequently may lead to error-prone decision-making. Another aspect to keep in mind is that people often do not always post their truly intentions, behaviors, opinions and ideas in social network sites (Manovich, 2012), which should be taken into account especially for human-centric studies.

Additionally, the establishment of standardized and well-documented methods to collect data from varied sources is of vital importance. González-Bailón et al. (2012) examined two communication networks based on the same population of Twitter messages but generated from distinct Twitter APIs (search and streaming). The authors found evidence of bias in the reconstructed networks partly due to different constraints and limitations in both APIs. Since researchers do not have full control of that source and the delivery of the sampled data, any subsequent analysis of the data may lead to distorted and unexpected results as the inconsistencies, errors and bias on the collected data may propagate to the data analysis itself.

For geospatial analyses, many studies limit themselves to geo-coded Tweets to avoid the additional challenge of geo-coding content. As a result, the retrieved data is a purposeful sample that may not representative of the (already biased) population of all Tweets. As commented earlier (González-Bailón et al., 2012), even when one single data source like Twitter is selected, it may become difficult if not impossible the integration and comparability of analysis outputs and findings, and hereby to some extent the reproducibility of the analysis too (Ostermann \& Granell, 2016).

Flickr has been used more at the beginning of our studied period ( 3 out of 4 publications are between 2008 and 2010) and declined later (1 in 2012). All but one study using Flickr and Twitter together were published recently (since 2013).

We observe a general trend in the analyzed papers in collecting large volumes of data in the order of millions of pieces of content, i.e. Tweets, images, and so forth (Fisher, 2012), in other words a focus on Big Crisis Data. Burns (2015) offers an important critique on Big Data and Digital Humanitarianism, pointing out that Big Data provides new practices, a distinct epistemology, and new social relations between customers of Big Crisis data (formal humanitarian sector and affected population), and providers of services (digital humanitarians). There is no evidence yet that the increasing volume of the data has led to additional insights. Indeed, as commented in Section 3, some studies employ data preparation and contextualization techniques to turn large VGI sets again into manageable, relevant and actionable data sets (Tsou \& Leitner, 2013). This again links to the issue of data representativeness, i.e. whether or not social data can be considered representative of the 
whole society (Roick \& Heusser, 2013), which also resembles to that of data quality versus quantity, would unavoidably require more attention in the future.

There is a notable absence of well-citied studies focusing on a systematic exploration of using OpenStreetMap in a disaster management context. This is all the more striking, as OSM has been used in practice to much greater effect in actual crisis response than any of the other data sources.

As for the analysis methods (Q4), there are no clearly discernible trends over time, with the majority of the studies being descriptive or exploratory in nature. To some extent, this is not surprising because exploratory experiments aim to discover correlations, but can rarely validate them. While correlations on Twitter data may be used as "predictors" earthquake early warning, this does not mean that earthquakes occur due to Twitter data. Causality is by far more challenging than other types of analysis. Indeed, it seems that a trend is increasingly taking shape which moves from hypothesis-driven analysis toward more data-driven analysis, where correlation is preferred (and often enough) over causality (Mayer-Schönberger \& Cukier, 2013).

For human-centric studies, SNA techniques are used to improve understanding on how a system behaves by constructing the networks from social data (e.g. Borgatti et al., 2013; Borgatti \& Ofem, 2010). SNA offers several techniques to understand a complex system like networks of people. Network properties such as betweenness, centrality, density, and degree describe the network's structure, whereas small worlds, scale free networks, and information diffusion models describe information flows through the network and thereby its behavior. When applied to disaster and emergency situations, these techniques let researchers discover the most relevant actors in a network and or to identify closed communities (clusters) in terms of highly connected nodes, for example. Again, many authors recently called for further research to put network concepts and social networks analysis into spatio-temporal data analysis (e.g., Crampton et al., 2013; Roick \& Heuser, 2013; Tsou \& Leitner, 2013; Stephens \& Poorthuis, 2015) as a hybrid approach to spatially study complex systems. A somewhat cross-cutting research theme is the development of "listener" tools to monitor social data social (restricted practically to Twitter) related to particular scenarios and/or topics, providing in some cases synchronized views in the form of a dashboard tool (MacEachren et al., 2011; Rogstadius et al., 2013).

\section{Conclusion and Outlook}

Our results suggest that much of the work in VGI4DM still concerns earlier phases of data analysis such as data preparation, i.e. merging, finding missing values, cleaning, annotating, filtering and so forth in order to make several data sets ready for the "true" analysis. This is 
reflected in the preponderance of data-centric studies and the fact that many of the humanand application-centric studies still put a secondary focus on data preparation and exploration. We argue also that VGI research is mature enough to move from purely data-centric objectives to more fundamental as well as applied research to leverage proper methodologies and analysis methods in VGI4DM scenarios.

Despite some exceptions like Rogstadius et al. (2013), most studies conducted off-line data analyses on historical data because real-time analysis of social media data aggravates many of the challenges. For example, Hurricane Sandy sparked more than 20 million "tweets" only in the course of the following five days (Shih, 2012), which requires cloud-based near real-time system architecture to process. There is still a lack of widely available real-time analytic tools to handle and process in real time vast amounts of data streams. This is independent of the persisting need to still validate the need for near real-time big data analysis. Likely, small data analysis of carefully collected samples has still its place in the methodological toolkit.

Most studies analyze social data over a short period of time. Obviously, the temporal dimension plays a key role if we want to study change over time. But the speed of change varies greatly between study objects: the impact of natural disasters (e.g. hurricane Sandy, Japan earthquake, etc.) may last days or weeks; the impact of social events (e.g. Arab Spring) may last weeks or even months. However, environmental change is often slower, sometimes over the course of several months, years or even decades. Getting a better understanding on how Earth and environmental processes change and evolve over longer periods of time ill improve understanding of natural disasters situations. Could VGI in conjunction with proper analytical tools help to understand such long timescale processes, or is it mostly suited for rapidly changing phenomena? Are citizen science projects with a stronger participatory character better suited to engage people in studies of long timescale events/phenomena that change slowly?

Most studies delimit their analysis to a small coverage area such as a city, districts within a city, and well-delimited areas like the surrounding of a quake epicenter. The main barrier to increasing spatial coverage seems to be the amount of data to be processed and analyzed. Are current VGI analysis toolkits ready to scale up to handle with large-scale (spatially) experiments? Imagine for example the case of studying spatial mobility patterns in Italy over a year using social media data. Can we conduct such an experiment with the analytical techniques for social media data available today?

Some scholars envision an emerging scientific field that would combine data science, GIScience and social sciences skills under the label of "location intelligence" (Wachowicz, 2013), with the ability to shape standardized methodologies to facilitate data collection, to carry 
out data analysis, and to compare the results beyond the eruption of popular technology and short-term research objectives. In this sense, emerging open source analytical tools, technology and easy access to public VGI sources are an undeniable opportunity for leveraging studies, analyses and novel purposes for VGI4DM beyond data collection. In order to ensure the validity, scope and impact of the results, they must fit hypothesis, research questions and overall analysis strategies (Leek \& Peng, 2015b). Some questions that need to be answered in the beginning of a VGI4DM analyses are: What kind of inferences we want to draw from the analysis? Are we interested in aggregate or individual effects? Are we interested in causal explanation or in making predictions? Are large quantities of data valid for studying individual effects as well as aggregate effects? What are the characteristics of an ideal VGI data set to address the goal of the study? Should there create a new metadata model for VGI to support reusable datasets and reproducible analysis? Which are the stakeholders of the analysis? We argue that many of these issues have not been addressed fully in many of the examined studies, where the research design follows often new, technology-driven opportunities, in some sense searching for the right lock to be opened with the new key, instead the other way around. This is unsurprising for a novel, rapidly evolving field of study. However, with soon a decade of research history, the study of VGI seems ready for these fundamental questions.

\section{References}

Abel F, Hauff C, Houben GJ, Stronkman R, Tao K (2012) Twitcident: fighting fire with information from social web streams. Proceedings of the 21st International Conference Companion on World Wide Web. ACM, New York, USA, pp 305-308

Adam NR, Shafiq B, Staffin R (2012) Spatial Computing and Social Media in the Context of Disaster Management. IEEE Intelligent Systems 27(6): 90-96

Andrienko G, Andrienko N, Bosch H, Ertl T, Fuchs G, Jankowski P, Thom D (2013) Thematic Patterns in Georeferenced Tweets through Space-Time Visual Analytics. Computing in Science and Engineering 15(3): 72-82

Antoniou V, Morley J, Haklay M (2010) Web 2.0 geotagged photos: Assessing the spatial dimension of the phenomenon. Geomatica: The Journal of Geospatial Information, Technology and Practice 64(1):99-110

Barrington L, Ghosh S, Greene M, Har-Noy S, Berger J, Gill S, Lin A, Huyck C (2012) Crowdsourcing earthquake damage assessment using remote sensing imagery. Annals of Geophysics 54(6)

Bengtsson L, Lu X, Thorson A, Garfield R, von Schreeb J (2011) Improved Response to Disasters and Outbreaks by Tracking Population Movements with Mobile Phone Network Data: A Post-Earthquake Geospatial Study in Haiti. PLoS Med 8(8): e1001083

Borgatti SP, Everett MG, Johnson JC (2013) Analyzing Social Networks. London: Sage Publications

Borgatti SP, Ofem B (2010) Overview: Social network theory and analysis. In A. J. Daly (Ed.), The ties of change: Social network theory and application in education. (pp. 1730). Cambridge, MA: Harvard Press 
Budak C, Georgiou T, Agrawal D, El Abbadi A (2013) GeoScope: Online Detection of GeoCorrelated Information Trends in Social Networks (2013). Proceedings of VLDB Endowment 7(4): 229-240

Burns R (2015) Rethinking Big Data in Digital Humanitarianism: Practices, Epistemologies, and Social Relations. GeoJournal 80 (4): 477-90.

Camponovo ME, Freundschuh SM (2014) Assessing Uncertainty in VGI for Emergency Response. Cartography and Geographic Information Science 41 (5): 440-55.

Chen F, Zhai Z, Madey G (2011) Dynamic adaptive disaster simulation: developing a predictive model of emergency behavior using cell phone and GIS data. In Proceedings of the 2011 Workshop on Agent-Directed Simulation. San Diego, CA, USA, pp 5-12

Casteleyn S, Garrigós I, Mazón JN (2014) Ten years of Rich Internet Applications: a Systematic Mapping Study, and beyond. ACM Transactions on the Web 8(3)

Chen H, Chiang RHL, Storey VC (2012) Business Intelligence and Analytics: From Big Data to Big Impact. MIS Quarterly 26(4):1165-1188.

Cheong F, Cheong C (2011) Social Media Data Mining: A Social Network Analysis Of Tweets During The 2010-2011 Australian Floods. PACIS 2011 Proceedings

Conover MD, Davis C, Ferrara E, McKelvey K, Menczer F, Flammini, A. (2013) The Geospatial Characteristics of a Social Movement Communication Network. PLoS ONE 8(3): e55957.

Corbane C, Lemoine G, Kauffmann M (2012) Relationship between the Spatial Distribution of SMS Messages Reporting Needs and Building Damage in 2010 Haiti Disaster. Natural Hazards and Earth System Science 12 (2): 255-65.

Corvey WJ, Vieweg S, Rood T, Palmer M (2010) Twitter in mass emergency: what NLP techniques can contribute. In Proceedings of the NAACL HLT 2010 Workshop on Computational Linguistics in a World of Social Media (WSA '10). Stroudsburg, PA, USA, pp 23-24

Craglia M, Ostermann F, Spinsanti L (2012) Digital Earth from vision to practice: making sense of citizen-generated content. International Journal of Digital Earth 5(5):398-416

Crampton JW, Graham M, Poorthuis A, Shelton T, Stephens M, Wilson MW, Zook M (2013) Beyond the geotag: situating 'big data' and leveraging the potential of the geoweb. Cartography and Geographic Information Science 40(2):130-139

Crandall DJ, Backstrom L, Cosley D, Suri S, Huttenlocher D, Kleinberg J (2010) Inferring social ties from geographic coincidences. PNAS 107(52): 22436-22441

Crawford K, Finn M (2015) The Limits of Crisis Data: Analytical and Ethical Challenges of Using Social and Mobile Data to Understand Disasters. GeoJournal 80 (4): 491-502.

Croitoru A, Crooks A, Radzikowski J, Stefanidis A (2013) Geosocial gauge: a system prototype for knowledge discovery from social media. International Journal of Geographical Information Science 27(12): 2483-2508

Crooks, A., Croitoru, A., Stefanidis, A., Radzikowski, J. (2013), \#Earthquake: Twitter as a Distributed Sensor System. Transactions in GIS, 17(1): 124-147

Curtis A, Mills JW (2012) Spatial video data collection in a post-disaster landscape: The Tuscaloosa Tornado of April 27th 2011. Applied Geography 32(2): 393-400

Cutter SL (2003) GI Science, Disasters, and Emergency Management. Transactions in GIS 7(4): 439-446

Davis Jr. CA, Pappa GL, de Oliveira DRR, de L. Arcanjo F (2011) Inferring the Location of Twitter Messages Based on User Relationships. Transactions in GIS 15: 735-751

de Albuquerque JP, Herfort B, Brenning A, Zipf A (2015) A Geographic Approach for Combining Social Media and Authoritative Data towards Identifying Useful Information for Disaster Management. International Journal of Geographical Information Science 29(4): 667-689

De Longueville B, Annoni A, Schade S, Ostlaender N, Whitmore C (2010) Digital Earth's Nervous System for Crisis Events: Real-Time Sensor Web Enablement of 
Volunteered Geographic Information" International Journal of Digital Earth 3 (3): 24259.

De Longueville B, Luraschi G, Smits P, Peedell S, de Groeve T (2010) Citizens as Sensors for Natural Hazards: A VGI Integration Workflow. Geomatica 64 (1): 355-63.

Diakopoulos N, de Choudhury M, Naaman M (2012) Finding and assessing social media information sources in the context of journalism. In Proceedings of the SIGCHI Conference on Human Factors in Computing Systems. ACM, New York, NY, USA, pp 2451-2460

Earle P (2010) Earthquake Twitter. Nature Geoscience 3(4): 221-222

Engström E, Runeson P (2011) Software product line testing - a systematic mapping study. Information and Software Technology 53(1): 2-13

Fisher F (2012) VGI as Big Data. GEO Informatics April/May 2012, pp 46-47

Foody GM (2014) Rating crowdsourced annotations: evaluating contributions of variable quality and completeness. International Journal of Digital Earth 7(8): 650-670

Gelernter J, Mushegian N (2011) Geo-parsing Messages from Microtext. Transactions in GIS 15(6): 753-773

Gelernter J, Balaji S (2013) An algorithm for local geoparsing of microtext. Geolnformatica 17(4): 635-667

Girardin F, Fiore FD, Ratti C, Blat J (2008) Leveraging explicitly disclosed location information to understand tourist dynamics: A case study. Journal of Location-Based Services 2(1): 41-54

Goldberg DW, Ballard M, Boyd JH, Mullan N, Garfield C, Rosman D, Ferrante AM, Semmens JB (2013) An evaluation framework for comparing geocoding systems. International Journal of Health Geographics 12(50)

González-Bailón S., Wang N, Rivero A, Borge-Holthoefer J, Moreno Y (2012) Assessing the Bias in Communication Networks Sampled from Twitter. arXiv:1212.1684

Goodchild M (2007) Citizens as Sensor: the world of volunteered geography. GeoJournal 69(4): $211-221$

Goodchild M, Li L (2012) Assuring the quality of volunteered geographic information of volunteered. Spatial Statistics 1: 110-120

Haworth B, Bruce E (2015) A Review of Volunteered Geographic Information for Disaster Management. Geography Compass 9, 237-250

Hobel H, Madlberger L, Thön A, Fenz S (2014) Visualisation of User-Generated Event Information: Towards Geospatial Situation Awareness Using Hierarchical Granularity Levels. In CEUR Workshop Proceedings, 1329:43-54.

Horita FE, Degrossi, LC, Assis LFG, Zipf A, de Alburquerque JP (2013) The use of Volunteered Geographic Information and Crowdsourcing in Disaster Management: a Systematic Literature Review. In Proceedings of 19th AMCIS. Chicago, USA, p 1-10

Horita FE., de Albuquerque JP, Degrossi LC, Mendiondo EM, Ueyama J (2015) Development of a Spatial Decision Support System for Flood Risk Management in Brazil That Combines Volunteered Geographic Information with Wireless Sensor Networks. Computers and Geosciences 80: 84-94.

Ikawa Y, Enoki M, Tatsubori M (2012) Location inference using microblog messages. In Proceedings of the 21st International Conference on World Wide Web. New York, NY, USA, pp 687-690

Intagorn S, Plangprasopchok A, Lerman K (2010) Harvesting Geospatial Knowledge from Social Metadata. In Proceedings of the 7th International ISCRAM Conference. Seattle, USA

Kalanteri M, Rajabifard A, Olfat H, Williamson I (2014) Geospatial Metadata 2.0 - An approach for Volunteering Geographic Information. Computers, Environment and Urban Systems 48:35-48

Kent JD, Capello Jr. HT (2013) Spatial Patterns and Demographic Indicators of Effective Social Media Content during the Horsethief Canyon Fire of 2012. Cartography and Geographic Information Science 40 (2): 78-89. 
King $\mathrm{G}$ (2011) Ensuring the data-rich future of the social sciences. Science 331:719-721

Leek JT, Peng RD (2015a) What is the question?. Science 347(6228): 1314-1315

Leek JT, Peng RD (2015b) Opinion: Reproducible research can still be wrong: Adopting a prevention approach. PNAS 112(6): 1645-1646

Levental S (2012) A New Geospatial Services Framework: How Disaster Preparedness Efforts Should Integrate Neogeography. Journal of Map \& Geography Libraries 8(2): 134-162

Liang Y, Caverlee J, Mander J (2013) Text vs. images: on the viability of social media to assess earthquake damage. Proceedings of the 22nd international conference on World Wide Web companion (WWW '13 Companion). International World Wide Web Conferences Steering Committee, Republic and Canton of Geneva, Switzerland, pp.1003-1006

Lingad J, Karimi S, Yin J (2013) Location extraction from disaster-related microblogs. In Proceedings of the 22nd international conference on World Wide Web. Republic and Canton of Geneva, Switzerland, pp 1017-1020

Liu W, Dong P, Liu S, Liu J (2014) A Rich Internet Application for Automated Detection of Road Blockage in Post-Disaster Scenarios. In Proceedings - 2014 IEEE Workshop on Advanced Research and Technology in Industry Applications, WARTIA 2014. doi:10.1088/1755-1315/18/1/012124.

Lue E, Wilson JP, Curtis A (2014) Conducting Disaster Damage Assessments with Spatial Video, Experts, and Citizens. Applied Geography 52: 46-54.

MacEachren AM, Jaiswal A, Robinson AC, Pezanowski S, Savelyev A, Mitra P, Zhang X, Blanford J (2011) SensePlace2: GeoTwitter analytics support for situational awareness. IEEE Conference on Visual Analytics Science and Technology (VAST), pp.181-190

Mandel B, Culotta A, Boulahanis J, Stark D, Lewis B, Rodrigue J (2012) A demographic analysis of online sentiment during hurricane Irene. In Proceedings of the Second Workshop on Language in Social Media. Association for Computational Linguistics, Stroudsburg, PA, USA, pp 27-36

Manovich L (2012) Trending: The Promises and the Challenges of Big Social Data. In: Gold $M(e d)$ Debates in Digital Humanities. Minnesota University Press

Mayer-Schönberger V, Cukier K (2013) Big Data: A revolution that will transform how we live, works and think. John Murray, London

McNamara L, Rohner C (2012) Mining conversations of geographically changing users. In Proceedings of the 21st International Conference companion on World Wide Web. New York, NY, USA, pp. 667-670

Meier P (2015) Digital Humanitarians: How Big Data is changing the face of humanitarian response. CRC Press, Boca Raton (FL)

Moon SP, Liu Y, Entezari S, Pirzadeh A, Papas A, Pfaff MS (2013) Top Health Trends: An information visualization tool for awareness of local health trends. In Proceedings of the 10th International ISCRAM Conference. Baden-Baden, Germany

Neis P, Zielstra D (2014) Recent Developments and Future Trends in Volunteered Geographic Information Research: The Case of OpenStreetMap. Future Internet 6:76-106

Ortmann J, Limbu M, Wang D, Kauppinen T (2011) Crowdsourcing Linked Open Data for Disaster Management. In Proceedings of the Terra Cognita Workshop on Foundations, Technologies and Applications of the Geospatial Web. Bonn, Germany

OGC SWE (2015) OGC's Sensor Web Enablement (SWE) standards http://www.opengeospatial.org/ogc/markets-technologies/swe

Ostermann FO, Granell C (2016) Advancing Science with VGI: Reproducibility and Replicability of Recent Studies using VGI. Transactions in GIS, doi 10.1111/tgis.12195

Panteras G, Wise S, Lu X, Croitoru A, Crooks A, Stefanidis A (2015). Triangulating Social Multimedia Content for Event Localization Using Flickr and Twitter: Triangulating Social Multimedia Content for Event Localization. Transactions in GIS 19(5): 694-715 
Petersen K, Feldt R, Mujtaba S, Mattsson M (2008) Systematic mapping studies in software engineering. 12th International Conference on Evaluation and Assessment in Software Engineering (EASE), pp 71-80

Pohl D, Bouchachia A, Hellwagner H (2012) Automatic Identification of Crisis-Related Subevents Using Clustering. In Proceedings of the International Conference on Machine Learning and Applications, pp. 333-338

Popoola A, Krasnoshtan D, Toth AP, Naroditskiy V, Castillo C, Meier P, Rahwan I (2013) Information verification during natural disasters. In Proceedings of the 22nd International Conference on World Wide Web. Republic and Canton of Geneva, Switzerland, pp 1029-1032

Poser K, Dransch D. (2010). Volunteered Geographic Information for Disaster Management with Application to Rapid Flood Damage Estimation. Geomatica 64 (1): 89-98.

Power R, Robinson B, Wise C (2013) Comparing web feeds and tweets for emergency management. In Proceedings of the 22nd International Conference on World Wide Web. Republic and Canton of Geneva, Switzerland, pp 1007-1010

Robinson B, Power R, Cameron M (2013) A sensitive Twitter earthquake detector. Proceedings of the 22nd international conference on World Wide Web companion (WWW '13 Companion). International World Wide Web Conferences Steering Committee, Republic and Canton of Geneva, Switzerland, pp. 999-1002.

Roick O, Heuser S (2013) Location Based Social Networks - Definition, Current State of the Art and Research Agenda. Transactions in GIS 17(5): 763-784

Rogstadius J, Teixeira C, Vukovic M, Kostakos V, Karapanos E, Laredo J (2013) CrisisTracker: Crowdsourced Social Media Curation for Disaster Awareness. IBM Journal of Research and Development 57(5): 4:1-4:13

Rutherford A, Cebrian M, Dsouza S, Moro E, Pentland A, Rahwan I (2013) Limits of social mobilization. Proceedings of the National Academy of Sciences of the USA 110(16): 6281-6286

Sagl G, Blaschke T, Beinat E, Resch B (2012) Ubiquitous Geo-Sensing for Context-Aware Analysis: Exploring Relationships between Environmental and Human Dynamics. Sensors 12, 9800-9822.

Santamaria E, Segor F, Tchouchenkov I (2013) Rapid Aerial Mapping with Multiple Heterogeneous Unmanned Vehicles. Proceedings of the 10th International ISCRAM Conference - Baden-Baden, Germany.

Scarletto EA (2014) Mapping the Literature of GIS. College \& Research Libraries 75(2): 179201

Schade S, Díaz L, Ostermann F, Spinsanti L, Luraschi G, Cox S, Nuñez M, De Longueville B (2013) Citizen-based sensing of crisis events: sensor web enablement for volunteered geographic information. Applied Geomatics 5(1): 3-18

Schelhorn SJ, Herfort B, Leiner R, Zipf A, De Albuquerque JP (2014) Identifying Elements at Risk from OpenStreetMap: The Case of Flooding. In Proceedings of the 11th International ISCRAM Conference, 508-12, University Park, USA

Schulz A, Paulheim H, Probst F (2012) Crisis Information Management in the Web 3.0 Age. In Proceedings of the 9th International ISCRAM Conference. Vancouver, Canada

Shelton T, Poorthuis A, Graham M, Zook M (2014) Mapping the Data Shadows of Hurricane Sandy: Uncovering the Sociospatial Dimensions of 'big Data.' Geoforum 52 (0): 16779

Shih G (2012) Twitter Activity Spiked During Hurricane Sandy: Over 20 Million Tweets About The Disaster This Week. The Huffington Post, 11/02/2012. http://www.huffingtonpost.com/2012/11/02/twitter-hurricane-sandy_n_2066281.html

Spinsanti L, Ostermann F (2013) Automated geographic context analysis for volunteered information. Applied Geography 43:36-44

Stefanidis A, Cotnoir A, Croitoru A, Crooks A, Rice M, Radzikowski J (2013) Demarcating new boundaries: mapping virtual polycentric communities through social media content. Cartography and Geographic Information Science 40(2): 116-129 
Steiger E, Porto de Albuquerque J, Zipf A (2016) An advanced systematic literature review on spatiotemporal analysis of Twitter data. Transactions in GIS, 19: 809-834

Stephens M, Poorthuis A (2015) Follow thy neighbor: Connecting the social and the spatial networks on Twitter. Computers, Environments and Urban Systems, 53: 87-95

Tatsubori M, Watanabe H, Shibayama A, Sato S, Imamura F (2012) Social web in disaster archives. In Proceedings of the 21st international conference on World Wide Web. New York, USA, pp 715-716

Tsou MH, Leitner M (2013) Visualization of social media: seeing a mirage or a message? Cartography and Geographic Information Science 40(2):55-60

Wang J, Pierce M, Yu M, Fox, G, Donnellan A, Parker J, Glasscoe M (2012) Using ServiceBased GIS to Support Earthquake Research and Disaster Response. Computing in Science \& Engineering 14(5): 21-30

Wirz M, Franke T, Roggen D, Mitleton-Kelly E, Lukowicz P, Tröster G (2013) Probing crowd density through smartphones in city-scale mass gatherings. EPJ Data Science 2:5

Wachowicz M (2013) New Frontiers for Geomatics. GIM International, 27(10)

Zhang C, Zhao T, Li W (2014) Towards an Interoperable Online Volunteered Geographic Information System for Disaster Response. Journal of Spatial Science 60(2): 257-275

Zielinski A et al. (2013) Social Media Text Mining and Network Analysis for Decision Support in Natural Crisis Management. In Proceedings of the 10th International ISCRAM Conference. Baden-Baden, Germany 